\title{
Editorial: Psychology and Experimental Philosophy
}

\author{
Joshua Knobe • Tania Lombrozo • \\ Edouard Machery
}

Published online: 1 December 2009

(C) Springer Science + Business Media B.V. 2009

Recent years have seen an explosion of new work at the intersection of philosophy and experimental psychology. This work takes the concerns with moral and conceptual issues that have so long been associated with philosophy and connects them with the use of systematic and well-controlled empirical investigations that one more typically finds in psychology. Work in this new field often goes under the name "experimental philosophy".

The aim of the present volume is to bring together some important new papers that demonstrate the promise and potential of this emerging field. The work collected here spans a wide range of philosophical topics - from ethics to epistemology to the philosophy of mind-and examines the ways in which empirical data can inform research in all of these fields. In addition, we have included a number of papers that offer critiques of contemporary experimental philosophy, suggesting ways in which existing studies might fail to address the philosophical questions they are supposed to be helping us to resolve.

Geoffrey Goodwin and John Darley present a series of experimental studies investigating people's ordinary intuitions in metaethics. Their research provides some tantalizing initial evidence that moral relativism is associated with a kind of "open" attitude toward ethical issues while a belief in objective moral truths is associated with a more "closed" attitude. Strikingly, the Goodwin and Darley studies show that relativism is more common among subjects who are high in an ability to

\footnotetext{
J. Knobe

Department of Philosophy, Yale University, 102 Conn. Hall, Department of philosophy, Yale University, PO Box 208306, New Haven, CT 06520, USA

e-mail: joshua.knobe@yale.edu

\section{T. Lombrozo}

Department of Psychology, Berkeley, 3210 Tolman Hall, Berkeley, CA 94720, USA

e-mail: lombrozo@berkeley.edu

E. Machery $(\bowtie)$

Department of History and Philosophy of Science, University of Pittsburgh, 1017 CL,

Pittsburgh 15260, USA

e-mail: machery@pitt.edu
} 
solve problems that involve considering alternative possibilities. Moreover, people who see their moral beliefs as objectively true are less likely to say that they would be willing to have someone who disagreed with them as a roommate, more likely to say that those who disagree with them have bad character traits, and less inclined to think that they might ever change their minds. The research outlined in this chapter initiates a new program of looking not just at what sort of attitude most people hold in the domain of metaethics but also at why different people might ultimately end up reaching quite different conclusions.

Eric Schwitzgebel and Joshua Rust take up the question as to whether moral reflection actually helps people to lead a moral life. As part of a systematic attempt to gain empirical insight into this question, they ask whether professors who specialize in moral and political philosophy are more likely to vote than are professors who specialize in other areas of philosophy. Strikingly, they find that a life spent examining moral and political questions does not appear to leave people any more likely to vote than does a life spent studying questions in metaphysics or epistemology. (In fact, there was even a non-significant trend whereby moral and political philosophers were somewhat less likely to vote than were other philosophers.) Especially in conjunction with Schwitzgebel and Rust's earlier work on the behavior of moral philosophers, this result raises serious questions about whether moral reflection actually does help people to live morally good lives.

Sandra Pellizzoni, Vittorio Girotto, and Luca Surian consider whether and how moral appraisals shape judgments of intentional action. In particular, how do an agent's beliefs influence whether the side effect of an intended action is itself judged to have been brought about intentionally? This question builds on a growing literature about the relationship between moral appraisals and theory of mind. In three experiments, the authors examine how foreknowledge of an action's side effects, false beliefs about an action's side effects, and an opportunity to hold an agent responsible influence judgments that a side-effect was brought about intentionally. Their findings suggest that participants rely on both evaluative and epistemic considerations in making judgments about intentional actions. Most surprisingly, they find that many participants are willing to say that an agent brought about a negative side effect intentionally, even when the agent was unaware that the side effect would occur! These findings point to the need for a more subtle analysis of the relationship between moral evaluation and theory of mind, with implications for both philosophy and psychology.

Andrew Monroe and Bertram Malle attempt to characterize the folk concept of free will. In contrast to what some philosophers have argued, for ordinary people, having free will does not require any commitment to a special form of nondeterministic causation or to the failure of determinism. Rather, for the folk, free will includes the capacity to choose so as to fulfill one's desires and the absence of internal and external constraints. The philosophical significance of this finding is well explained by Monroe and Malle. If no metaphysical assumption is built into the folk concept of free will, then scientific determinism should not threaten people's belief in free will and the related belief in people's responsibility for their moral and immoral actions.

Bryce Huebner, Michael Bruno, and Hagop Sarkissian examine whether people think similarly about consciousness in Western and Eastern cultures. Specifically, 
they examined whether Americans and Chinese judge that group agents, such as corporations (think: General Motors or Apple), can have conscious feelings and emotions: for instance, would it make sense to say that General Motors feel fear? Although both Chinese and Americans were more reluctant to ascribe conscious feelings and emotions to individuals than to corporations (as Knobe and Prinz had already shown), they found that Chinese did not distinguish group agents from individuals as strongly as Americans: Chinese were almost as willing to ascribe conscious feelings and emotions to group agents and to individuals. Huebner, Bruno, and Sarkissian also argue that this finding has substantial philosophical implications: it suggests that some of the most well-known intuitions in the philosophy of mind might not be particularly convincing for Easterners.

Matthew Weber and Daniel Osherson tackle the nature of inductive reasoning, a classic problem for both philosophy and cognitive psychology. Inspired by Hume, they develop an account of inductive inference grounded in similarity. Consider a problem like those encountered by participants in their experiment: given that bears have at least $18 \%$ of their cortex in the frontal lobe, how likely is it that camels do? Their account suggests that this probability estimate will depend on the perceived similarity between bears and camels. This approach is extended to deal with more complex inferences, but the most innovative step comes in their measure of similarity. Weber and Osherson utilize functional magnetic resonance imaging (fMRI) to probe the neural representations underlying categories like "bear," and "camel," and develop a measure of shared neural representation as an indicator of similarity. This approach can potentially side-step a perennial concern for more common measures of similarity: that participants use the inductive relationship between two categories as a measure of similarity, and not the reverse. Using their model of inductive inference and their neural measure for similarity, they predict participants' probability judgments with high accuracy. While the authors acknowledge that this supports a descriptive re-interpretation of Hume's views about similarity and inductive inference, and doesn't address questions about the justification for induction, the contribution is an elegant example of how philosophical, psychological, and formal approaches can be mutually informative.

Joshua May, Walter Sinnott-Armstrong, Jay Hull and Aaron Zimmerman take up the study of people's intuitions about knowledge. Philosophers working on this topic have often suggested that people's intuitions can be affected by either (a) the practical interests at stake or (b) the alternatives that have been made salient. Though different philosophers have proposed radically different explanations for these effects, philosophers of a wide range of persuasions have agreed that the effects do, in fact, exist, and philosophers with otherwise quite different views have agreed that the effects can be observed in one particular example that involves two people waiting in line at a bank. May and colleagues presented subjects with that exact case. Surprisingly, the predicted effect did not emerge. Instead, people said that the character in the story did have knowledge even in precisely the case that philosophers of these opposing views had agreed would not be counted as knowledge. This result raises serious questions for future research in epistemology.

Simon Cullen presents a trenchant critique of one of the most pervasive methods in experimental philosophy: the use of surveys to solicit intuitions. With the rise of experimental philosophy, undergraduates, park-strollers, and Internet-users around 
the world have been confronted with wayward trolley-cars, fanciful tales about Gödel, and a colorful assortment of epistemic conundrums. Cullen argues that the judgments elicited by such cases are influenced by a number of factors, including the pragmatic features of the survey and the context. As a result, participants' responses to surveys cannot be taken as direct reflections of their folk intuitions about the philosophical cases. Cullen draws on previous work from psychology on survey methodology to develop this critique, and illustrates his points with empirical studies of epistemic intuitions. The paper poses a challenge that new work in experimental philosophy will have to meet with the use of more sophisticated survey methodology, more nuanced interpretations of survey data, and a greater reliance on alternative methods.

Having distinguished two different approaches in experimental philosophy- "the negative program" and "the positive program"-Joshua Alexander, Ron Mallon, and Jonathan Weinberg scrutinize the prospects of the positive program. They consider four ways of articulating the positive program (viz. direct extramentalism, semantic mentalism, conceptual mentalism, and mechanist mentalism), and, for each of these four articulations, they systematically identify some striking difficulties. Their conclusion is pessimistic: according to them, the prospects of the positive program of experimental philosophy look bleak. No doubt, those experimental philosophers who endorse the positive program partly or entirely (including the three of us!) will have to reckon with these objections, and they will have to explain why experimental methods are well tailored to meet their goals.

The collection of papers brought together in this volume reflects the diversity of experimental philosophy. The methods range from behavioral lab experiments to brain imaging to analyses of voting records. And the topics are equally diverse, mirroring the plethora of philosophical issues amenable to input from empirical investigation. This volume also reflects the fact that experimental philosophy is a developing field - one engaged in the process of examining its own foundations. By generating exciting new work pushing the frontiers of experimental philosophy, and continuing to engage in the critical examination of foundational issues, we anticipate a promising future for the intersection of experimental psychology and experimental philosophy. 